\title{
Blurred Face Recognition Algorithm Guided by a No-Reference Blur Metric
}

\author{
Cécile Fiche ${ }^{a}$, Patricia Ladret ${ }^{a}$ and Ngoc-Son $\mathrm{Vu}^{a}$ \\ ${ }^{a}$ Département Images et Signal, Gipsa-Lab, 961 rue de la Houille Blanche - BP 46, 38402 \\ Grenoble, France;
}

\begin{abstract}
Performance of face recognition systems drop drastically when blur effect is present on facial images. In this paper, we propose a new approach for blurred face recognition. Our method is based on a measure of the level of blur introduced in the image using a no-reference blur metric. The face recognition process can be performed with any facial feature descriptor to allow the combination of alternative methods of overcoming data acquisition problems introduced in an image. To assess its efficiency, the approach has been applied with Gabor wavelets, Local Binary Patterns (LBP) and Local Phase Quantization (LPQ) facial descriptors on the FERET data-set. Experimental results clearly show the strength of this method to various forms of blur whatever the facial feature descriptor algorithm implemented.
\end{abstract}

Keywords: Face recognition, Blur, No-Reference Metric, Computer vision

\section{INTRODUCTION}

During the last few decades, automatic recognition of human faces has been developed into a highly active field of research. Multiple commercial applications have been designed such as surveillance, security and access control systems. Enormous investment has been made to ensure public safety in most common infrastructures, industries and institutions. Especially video surveillance systems have become one of the most popular systems in terms of security. The greatest number offer intelligent analysis software especially based on faces identification algorithms. Such a system acquires facial images in uncontrolled areas so that it does not introduce any constraints for the subjects resulting in their changes in behaviour. This makes the video surveillance system very attractive as it can be used efficiently to prevent trouble makers from entering security areas. However, facial identification algorithms have a completely satisfactory recognition rate only when images processed by the system are full-face images with good quality. Uncontrolled conditions of acquisition (lighting conditions, blur effects, blocking effects, large changes in facial pose) interfere with images analysis and performances drop drastically. Nonetheless, millions of video surveillance cameras are installed every year, but real-time information provided is still underexploited or lost as no reliable automatic identification system exists. Consequently, most research has been focused on alternative methods of overcoming data acquisition problems principally caused by large changes in facial pose and lighting conditions. ${ }^{1}$ Little research concerning blur effect has been carried out until now, even though blur is a key factor leading to the degradation of image quality. Most of the blur effect occurs when the camera or the subject moves while the shutter is open or when the camera is out-of-focus. In such conditions, most face identification algorithms are not sufficiently robust leading to a very low recognition rate. This is such a recurrent problem in video surveillance that many blurred images can not provide any information about a face. In this paper, the effect of various types and levels of blur on face identification is considered. Until now, various methods have been proposed to deal with the recognition of blurry images. One way is to restore the images before the identification stage but this introduced new artefacts to the image. ${ }^{2}$ To deal with this problem, Stainvas and Intrator ${ }^{3}$ proposed a hybrid recognition-reconstruction network. However, to make the network process efficient and consistent with different forms of blur, the system needs to expand the gallery data-set with blurry images for which the degradation transformation is known. Consequently, the system involves some a priori knowledge about the degradation model. In face analysis, a classical approach is to find a face descriptor highly robust to a particular disturbance. Consequently, another way is to find a feature vector for face appearances such that blur deformation can be compensated for. This problem has been addressed efficiently using a blur insensitive operator ${ }^{4}$ with the Local Phase Quantization (LPQ) method ${ }^{5}$. However, in video surveillance applications, various problems can be encountered on the same image such as blur and pose 
changes. In such cases, identification becomes almost impossible; until now, there has been no face descriptor sufficiently robust to different acquisition problems giving a good recognition rate. In this work, we introduce a new approach for blurred face identification based on a no-reference blur metric (BluM) ${ }^{6}$ We used this metric to adapt the face identification algorithm to the input image according to its level of blur. This approach can be combined to any face feature vector while still considering blur effect. Consequently, with our approach, it is possible to choose the most appropriate face feature extraction method according to the disturbances introduced in the input face images.

To test the robustness of our approach against different forms of blur, we artificially blurred images with a Gaussian and a Motion filter. We tested our approach with three different methods in order to assess its reliability. In the first part of this article, we will briefly present Gabor, LBP and LPQ methodology. The BluM metric will be introduce in the third part and our approach will be described in section 4. Finally, we will explained our experiments and discuss results obtained in section 5 and 6 .

\section{FACE DESCRIPTION BASED ON GABOR WAVELETS, LBP AND LPQ FEATURES}

\subsection{Facial features extraction using Gabor wavelets}

A spatial-frequency representation is advantageous to detect particular features in an image as it is an efficient way to minimise illumination and pose variation. ${ }^{1}$ Therefore wavelet transformations turn out to be very efficient in extracting facial features. It has been shown that Gabor filters fit the 2-D spatial response of simple cells in the visual cortex and that they minimise the Heisenberg uncertainty principle by providing the best trade-off between space and frequency resolution in 2-D domains. ${ }^{7}$ Therefore, Gabor filters exhibit relevant properties from both biological and mathematical points of view for feature face extraction. The 2-D Gabor wavelets generated by rotation and dilatation from the mother Gabor wavelet is defined as follows: ${ }^{2}$

$$
\psi_{\mu, \nu}(z)=\frac{\left\|k_{\mu, \nu}\right\|^{2}}{\sigma^{2}} e^{\frac{-\left\|k_{\mu, \nu}\right\|^{2}\|z\|^{2}}{2 \sigma^{2}}}\left(e^{i k_{\mu, \nu} z}-e^{-\frac{\sigma^{2}}{2}}\right) .
$$

Where $\mu$ ans $\nu$ are the orientation and scale of the Gabor filter respectively and $k_{\mu, \nu}$ is the wave vector defined as follows:

$k_{\mu, \nu}=k_{\nu} e^{i \theta_{\mu}}$ where $k_{\nu}=\frac{f_{\max }}{\sqrt{2}^{\nu}}$ and $\theta_{\mu}=\frac{\pi \mu}{8}$.

$f_{\max }$ is the maximum frequency and $\sqrt{2}$ is the spacing factor between different central frequencies. Finally the Gabor wavelet representation of a face at orientation $\mu$ and scale $\nu$, is obtained by convolving the facial image with the corresponding Gabor filter. The sample image is usually represented by a set of 40 Gabor wavelets ${ }^{8,9}$. In this work, we used a set of 40 Gabor kernels with 8 orientations $\mu=\{0,1, \ldots, 7\}$ and 5 different scales $\nu=$ $\{0,1, \ldots, 4\}$.

\subsection{Facial features extraction using Local Binary Patterns (LBP)}

The LBP texture analysis operator was first introduced by Ojala et al. ${ }^{10}$ For each pixel of an image, the operator considers a neighbourhood of size $3^{*} 3$ and labels the 8 neighbours with a binary number. To do so, the centre value is used as a threshold. Each pixel value above is equal to one and zero otherwise. The an histogram of the labels is made and used as a texture descriptor. Later, two others extension of the original operator have been done. ${ }^{11}$ The method has been first extended to circular neighbourhoods of different radius sizes to detect larger patterns. Then the so-called uniform LBP has been introduced. Considering the binary code as circular bit string, all the strings containing at most two bitwise transitions (0-1 or 1-0) are called uniform. Uniform patterns contain most of the texture information while they represent only 58 of the 2568 -bit patterns. In recent year, T. Ahonen ${ }^{12}$ introduced a powerful face recognition algorithm based on LBP texture descriptor. It is an efficient descriptor that stands up to illumination changes and outperforms most of the face recognition methods. ${ }^{12}$ The facial image is first divided into m regions $\left\{R_{0}, R_{1}, R_{m-1}\right\}$. For each of them, the LBP operator is applied and a histogram of the labels is made in order to obtain local facial feature descriptors. The global representation of the face is obtained by combining all the local descriptors. In this work, we used the $L B P_{P, R}^{u_{2}}$ operator with $P=8$ and $R=2$ and we divided the face image into $8^{*} 8$ regions. $P$ refers to the number of 
sampling points equally spaced on a circle of radius $R$ and $u 2$ stands for using only uniform patterns. Finally, a chi-squared distance is used to compare feature vectors of a sample image with those of the gallery data-set.

\subsection{Facial features extraction using Local Phase Quantization (LPQ)}

The blur insensitive LPQ texture descriptor was first introduced by Ojansivu and al. ${ }^{5}$. A two dimensional Discrete Fourier transforms (2D-DFT) defined as follows is performed over a rectangular neighbourhood $N_{z}$ at each pixel position $\mathrm{z}=(\mathrm{x}, \mathrm{y})$ of the image $\mathrm{I}$.

$$
F(u, z)=\sum_{w \in N_{z}} I(z-w) e^{-i 2 \pi u^{T} w} .
$$

The 2D-DFT is computed at only four frequency points $u_{i}$ where the phase of the DFT is shown to be invariant to centrally symmetric blur ${ }^{5}: u_{1}=[a, 0], u_{2}=[0, a], u_{3}=[a, a]$ and $u_{4}=[a,-a] . a$ is a scalar choose to satisfy this condition. Then the sign of the real and the imaginary part of each Fourier coefficient is extracted resulting in a binary coefficient $q_{j}(\mathbf{x})$ :

$$
q_{j}(\mathbf{x})=\left\{\begin{array}{ll}
0 & \text { if } g_{j}(\mathbf{x}) \geq 0 \\
1 & \text { otherwise }
\end{array}\right\} .
$$

where $g_{j}(\mathbf{x})$ is the jth component of the vector $G_{x}=[\operatorname{Re}\{F(u, z)\}, \operatorname{Im}\{F(u, z)\}]$

The eight binary code obtained gives the phase information. Finally a label image $F_{L P Q}$ is obtained by representing the binary code as integer values between 0 and 255 .

$$
F_{L P Q}(\mathbf{x})=\sum_{j=1}^{8} q_{j}(\mathbf{x}) 2^{j-1}
$$

In recent year, Ahonen ${ }^{4}$ introduced a powerful face recognition algorithm based on LPQ texture descriptor. In the same way as the LBP method, the LPQ methodology can be summarized into four steps. First of all, the LPQ operator is applied on the input image to get the label LPQ image. Then this label image is split into small regions. For each of them, an histogram of the labels is made to obtain local facial feature descriptors. The global LPQ representation of the face is obtained by combining all the local feature descriptors. In this work, we used the same parameters as those introduced in the paper of Ahonen et al. ${ }^{4}$

\section{BLUR METRIC (BLUM)}

The BluM metric ${ }^{6}$ approach is based on the human blur perception. Human visual system perceives differences between blurred and sharp image but can not accurately discern a blurred image with the same re-blurred image. In terms of gray level, this means that a blurry process introduces strong intensity variations between neighbouring pixels of a sharp image whereas only slight intensity variations between neighbouring pixels are introduced if the image is already blurred. Based on this observation, the Blur Metric uses the blur discrimination properties of the human perception to create a reference image for blur estimation. To do so, a strong low-pass filtering process along both horizontal and vertical directions is applied on the original picture $F$ and the blurred images $B_{H}$ and $B_{V}$ obtained are used as reference. The absolute difference intensity between neighbouring pixels is computed along both horizontal and vertical directions for the original image and the references to get the absolute difference images.

$$
\begin{gathered}
d F_{V e r}=A b s(F(i, j)-F(i-1, j) ; 1 \leq i \leq m-1,0 \leq j \leq n-1 \\
d F_{H o r}=A b s(F(i, j)-F(i, j-1) ; 1 \leq j \leq n-1,0 \leq i \leq m-1 \\
d B_{V}=A b s\left(B_{V}(i, j)-B_{V}(i-1, j) ; 1 \leq i \leq m-1,0 \leq j \leq n-1\right. \\
d B_{H}=A b s\left(B_{H}(i, j)-B_{H}(i, j-1) ; 1 \leq j \leq n-1,0 \leq i \leq m-1\right.
\end{gathered}
$$


Then, variations from the initial picture are computed:

$$
\begin{gathered}
V_{V e r}=\operatorname{Max}\left(0, d F_{V e r}(i, j)-d B_{V e r}(i, j) ; 1 \leq i \leq m-1,0 \leq j \leq n-1\right. \\
V_{H o r}=\operatorname{Max}\left(0, d F_{H o r}(i, j)-d B_{H o r}(i, j) ; 1 \leq j \leq n-1,0 \leq i \leq m-1\right.
\end{gathered}
$$

If variations are important, it means that the original picture is sharp whereas slight variations means that the original picture was already blurred. To compared those variations, a sum of each distance $d F_{V e r}, d F_{H o r}$, $d V_{V e r}$ and $d V_{H o r}$ is computed as follows:

$$
\begin{aligned}
S F_{V e r} & =\sum_{i=1}^{m-1} \sum_{j=1}^{n-1} d F_{V e r}(i, j) \text { and } S F_{H o r}=\sum_{i=1}^{m-1} \sum_{j=1}^{n-1} d F_{H o r}(i, j) . \\
S V_{V e r} & =\sum_{i=1}^{m-1} \sum_{j=1}^{n-1} d V_{V e r}(i, j) \text { and } S V_{H o r}=\sum_{i=1}^{m-1} \sum_{j=1}^{n-1} d V_{H o r}(i, j) .
\end{aligned}
$$

Finally a normalisation is made to obtain a no-reference perceptual blur estimation ranging from 0 to 1 , respectively the best and the worst quality in term of blur perception:

$$
b_{-} F_{V e r}=\frac{S F_{V e r}-S V_{V e r}}{S F_{V e r}} \text { and } b_{-} F_{H o r}=\frac{S F_{H o r}-S V_{H o r}}{S F_{H o r}} \text {. }
$$

The final blur value, ${b l u r_{F}}$, corresponds to the maximum value between $b_{-} F_{V e r}$ and $b_{-} F_{H o r}$ :

$$
\operatorname{blur}_{F}=\operatorname{Max}\left(b_{-} F_{V e r}, b_{-} F_{H o r}\right) .
$$

\section{OUR APPROACH}

A face recognition system must recognised a face among a list of known persons. Consequently automatic recognition systems must compare a facial image acquired with a video camera to a pre-defined data-set (gallery) containing facial images of known persons. Thus, the system can suggest a classification for face recognition. In face analysis, a classical approach is to find a feature descriptor for face representation. Thus the classification is done by comparing the feature vector of the input image with each feature vector of the gallery. However, the gallery is usually made of good quality images contrary to the input face to be recognised such that face feature vectors obtained for both are not constructed with the same characteristics. In this paper, we propose a novel recognition approach to adapt each facial feature vector of each image of the gallery to the one of the image test. This method is divided into a pre-processing step and a recognition step. The pre-processing step is a degradation process. It is designed to filter out the over-much information contained in images of the gallery to save the most relevant facial features according to various levels of blur. The recognition step uses the Blur metric to evaluate the level of blur of the input image and adapts the gallery to get the best recognition rate. This is a low complexity and highly effective approach as it can be applied with various face descriptors and without any a priori knowledge about the blur.

\subsection{Pre-processing step}

For the degradation step, we choose a Gaussian filter which is an easy way to implement a low pass filtering. Thus all the images were convolved with a $F g_{(s, h)}$ filter. $F g_{(s, h)}$ refers to a rotationally symmetric Gaussian filter with a standard deviation $s=\{0,0.5,1,1.5, \ldots, 5\}$ and a square matrix of size $h=\{3,5,7,9,11\}$. According to the parameters $(\mathrm{h}, \mathrm{s})$, the original gallery $T_{(0,0)}$ was split into 50 others galleries $T_{(s, h)}$. Each of them corresponding to the original data-set images convolved with a given filter $F g_{(s, h)}$. Finally, we used the BluM metric to allocate a coefficient to each gallery.

To do so, we used the BluM to get a no-reference perceptual blur estimation $b i_{(s, h)}$ for each blurry image $I_{(s, h)}$ 


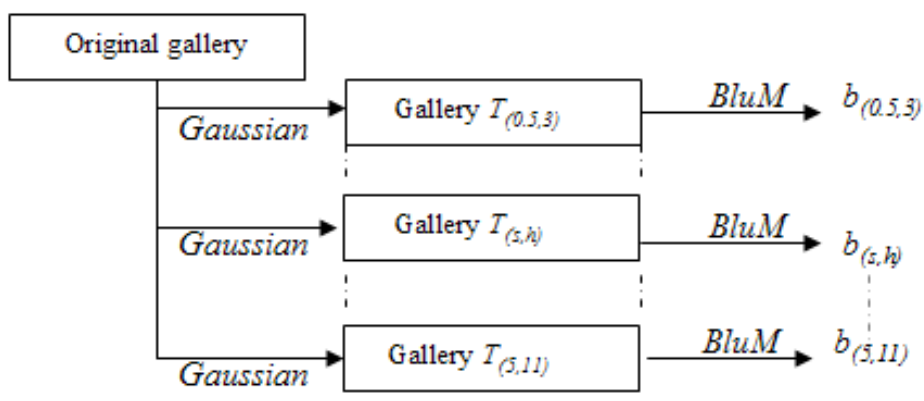

Figure 1. Flow-chart of the pre-processing step.

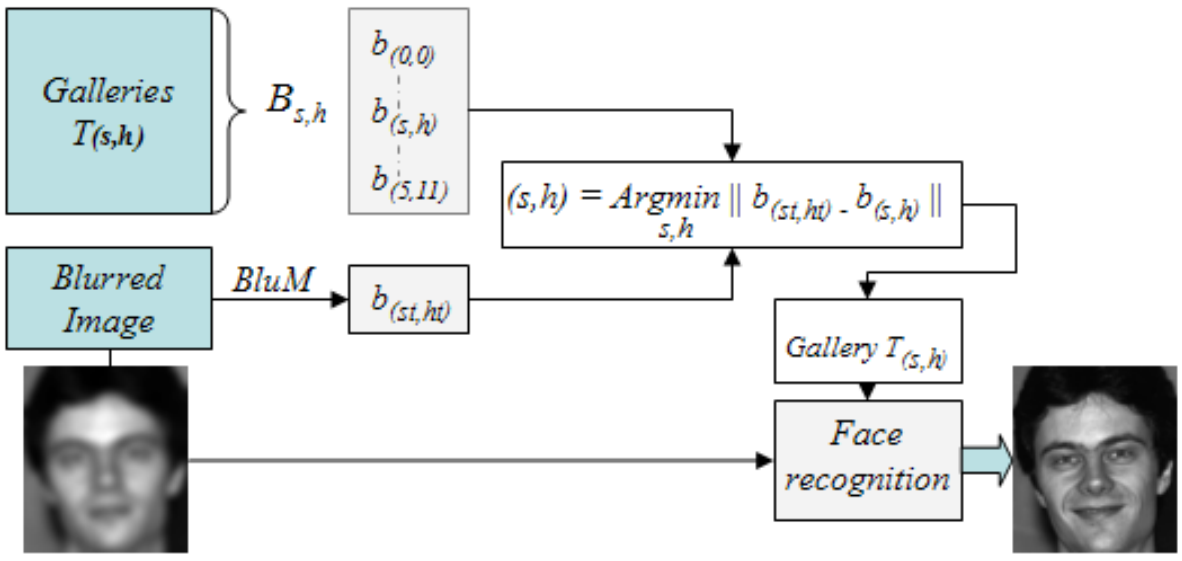

Figure 2. Flow-chart of the blurred face recognition approach guided by a no-reference blur metric.

of a given $T_{(s, h)}$ data-set. Then, we average this set of coefficients and allocate the mean scalar value obtained, $b_{(s, h)}$, to the gallery $T_{(s, h)}$. Finally we got a vector $B_{s, h}=\left[b_{(0,0)}, b_{(0.5,3)}, b_{(1,3)}, \ldots, b_{(5,11)}\right]$ containing all the scalar values corresponding to each data-set $T_{(s, h)}$. Parameters $(s, h)=(0,0)$ refers to the original gallery with no degradation. Notice that this pre-process step must performed only once and can be obtained in advance. A flow-chart of the pre-processing step is given in Fig. 1.

\subsection{Blurred faces recognition}

We first try to estimate the strength of the degradation caused by blur on the tested image. To do so, we use the aforementioned blur metric and we get a no-reference perceptual blur estimation ranging from 0 to 1 . This coefficient $b_{t}$ is allocated to any input image according to the strength of degradation whatever the form of blur considered. Then, this estimation value is compared to all the coefficients $b_{(s, h)}$ already computed during the pre-process step. To do so, the L2 norm $\left\|b_{t}-b_{(s, h)}\right\|$ is calculated for each coefficient $b_{(s, h)}$. The minimum is obtained for the given parameters

$$
(s, h)=\underset{s, h}{\arg \min }\left\|b_{t}-b_{(s, h)}\right\|
$$

which set the gallery to be used for the recognition. Finally, facial feature vectors are obtained and compared with already proposed face recognition algorithms. A flow-chart of the approach is given in Fig. 2.

\section{RESULTS}

\subsection{Data-set description}

We used a data set from the FERET database ${ }^{13}$ to assess the efficiency of the proposed new recognition approach for blurred faces. The FERET image body consists of 14051 eight-bit greyscale images of human head. 

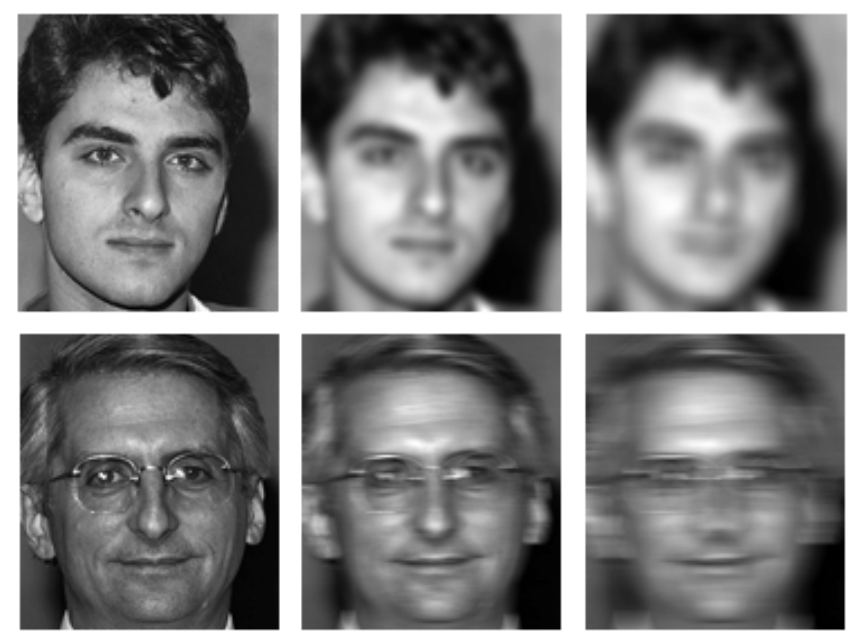

Figure 3. Upper row from left to right: original picture, original picture blurred with a Gaussian filter $F g_{(2,11)}$, original picture blurred with a Gaussian filter $F g_{(5,11)}$. Bottom row from left to right: original picture, original picture blurred with a Motion filter $F m_{(7,0)}$, original picture blurred with a Gaussian filter $F m_{(17,0)}$.

Specifically, we used $1194 * 2$ frontal images resulting from the fa (regular facial expression) and fb (alternative facial expression) probe set. Performance of our approach has been tested on images from the fb probe set. Each of them has been artificially blurred with two different filters $F g_{(s, h)}$ and $F m_{(p, t)}$. As described in the previous section, $F g_{(s, h)}$ refers to a rotationally symmetric Gaussian filter with a standard deviation $s=\{1,2,3,4,5\}$ and a square matrix of size $h=\{3,5,7,9,11\}$. Consequently, we tested our approach with 25 galleries corresponding to 25 forms of gaussian blur. Each gallery containing 1194 images. $F m_{(p, t)}$, once convolved with an image, is a model of the blur occurring when the camera is moving of $p=\{7,9,11,13,15,17\}$ pixels with an angle of $t=\{0,45,90,135\}$ degrees in relation to the subject. Similarly, we tested our approach with 24 galleries corresponding to 24 forms of motion blur. Each gallery containing 1194 images. Examples of two original images and the corresponding blurred version are shown in Fig. 3.

\subsubsection{Experiments}

In this work, two experiments have been carried out to assess the performance of our approach against different forms of blur. In the first one, we artificially blurred images with a Gaussian filter. In the second one we used a motion filter to model the blur occurring when the camera is moving. For each experiment, we tested our approach with three different methods in order to assess its reliability. First of all, we applied our new approach to the Gabor wavelets and the Local Binary Pattern (LBP) face descriptor. It was interesting to test our approach with such methods not only because they are the most common used in face recognition but the feature descriptor they compute is not blur invariant. Both of them exhibit relevant properties to extract insensitive features due to various distortions such as illumination, pose and changes in expression. However, given that the main cause of blur is due to a low pass operation, such facial features do not stand up to blur effect. The Gabor filter acts as a band-pass filter, consequently, Gabor wavelet representation of blurred images is strongly sensitive to both form and strength of the degradation. In the same way, in the spatial domain, a blurry process leads to slight variations between neighbouring pixels. Therefore as LBP method is a local texture descriptor based on neighbourhood pixel values, it is not a blur invariant feature descriptor either. In a second time we used the $\mathrm{LPQ}^{4}$ descriptor as it is highly tolerant of blur variation. In this paper, only results obtained for the Gaussian filter $F g_{(s, 11)}$ with increasing standard deviation $s=\{0,1,2,3,4,5\}$ and the Motion filter $F m_{(p, 0)}$ with increasing number of pixels $p=\{7,9,11,13,15,17\}$ are shown. Both correspond to the strongest blur we applied on images. 


\subsection{Results analysis}

\subsubsection{Experiment 1}

The recognition rate obtained for tested images artificially blurred with the Gaussian filter $F g_{(s, 11)}$ for Gabor, LBP and LPQ are shown in Fig. 4. As it can be seen in the results, our approach highly improves the recognition performance of blurry images when using Gabor, LBP or LPQ features. When blur is increasing, the recognition rate obtained with Gabor filters drops drastically from $80.9 \%$ to $4.52 \%$ whereas the one obtained with LBP drops from $95.64 \%$ to $53.10 \%$. Consequently, LBP is more tolerant to blur than Gabor wavelets but still very sensitive. When we adapt the gallery to the images tested, a much lower decrease is observed. With our approach combined to the Gabor filters, the recognition rate obtained decreases until 59.97\%. As LBP is slightly more tolerant to blur, the results we obtained give very good recognition rates even for the strongest blur we applied. So, we achieved a very good recognition rate approximately constant varying between $97.32 \%$ and $96.15 \%$. Nonetheless, we obtained the best recognition rate with the LPQ feature descriptor. The combination of our approach with such descriptors produces very good results whatever the strength of blur applied. So, we got a recognition rate approximately constant varying only between $97.82 \%$ and $96.48 \%$. Similar results have been achieved with tested images artificially blurred with lower strength Gaussian filters.

\subsubsection{Experiment 2}

In this experiment, we achieved the recognition rate for tested images artificially blurred with the Motion filter $F m_{(p, 0)}$. Results are illustrated in Fig. 4. Similar to the previous experiment, we tested our approach with Gabor, LBP and LPQ feature descriptors. Similar results have been achieved. As shown in Fig. 4, from $p=9$ onwards, our approach enhances the recognition rate of the face recognition algorithm based on Gabor feature descriptor. We also tested our approach with the Motion filters $F m_{(3,0)}$ and $F m_{(5,0)}$. The recognition rate obtained with our approach was $84.42 \%$ for $p=3$ and $79.90 \%$ for $p=5$ against $85.01 \%$ and $81.49 \%$ for $p=3$ and $p=5$ respectively without our approach. Consequently, our approach seems to be less efficient for low strength motion filter but the drop is lower than $1 \%$. We also applied our approach to LBP and LPQ with such filters. Our approach always enhances the recognition rate, even with $F m_{(3,0)}$ and $F m_{(5,0)}$ filters. Nonetheless, we did not put our results as the variations was negligible compared to those obtained for stronger filter. Without our approach, the recognition rate obtained with the LBP descriptor clearly decreases with increasing number of pixels until 67.34\%. With the LPQ descriptor, we observed a decrease until 85.26\%. Consequently, LPQ method is still tolerant of blur variation but it seems more robust to Gaussian blur than Motion blur. With our approach, we achieved a very good recognition rate approximately constant varying between $95.98 \%$ and $93.30 \%$ with LBP feature descriptor and a recognition rate varying between $96.90 \%$ and $93.13 \%$ with LPQ feature descriptor. Consequently, results obtained assess the efficiency of our approach against different forms of blur.

\section{DISCUSSION AND CONCLUSIONS}

In this paper, we have proposed a new blurred face identification approach which can be combined with different facial feature vector algorithms known to be invariant to different forms of acquisition problem. The effectiveness of this approach, based on a no-reference blur metric, has been successfully tested with three of the most efficient descriptors for face recognition. This face identification approach has been tested on images using a data set from the FERET database. Experimentally, results clearly showed the strength of this approach to different types of blur whatever the descriptor for face appearance considered. Usually, input images are compared to a pre-defined data-set formed with good quality images. However a blurry process leads to a low pass operation. Consequently, as the feature face descriptor is applied on input images and on images from the gallery data-set, face feature vectors obtained for both are not constructed with the same characteristics. In this approach, the metric provides information about the level of blur introduced in the input image. Consequently, it enables the detection of the relevant frequencies for face identification and to alter the training data-set thereof. This type of approach avoids a restoration process which introduces new features on images while the degradation process is carried out in a pre-processing step to enable real-time recognition applications such as video-surveillance for instance. Indeed, time of the recognition step is only limited by the facial feature vectors implemented. In this work, this approach was applied to the face recognition problem but it can be used also in other recognition 

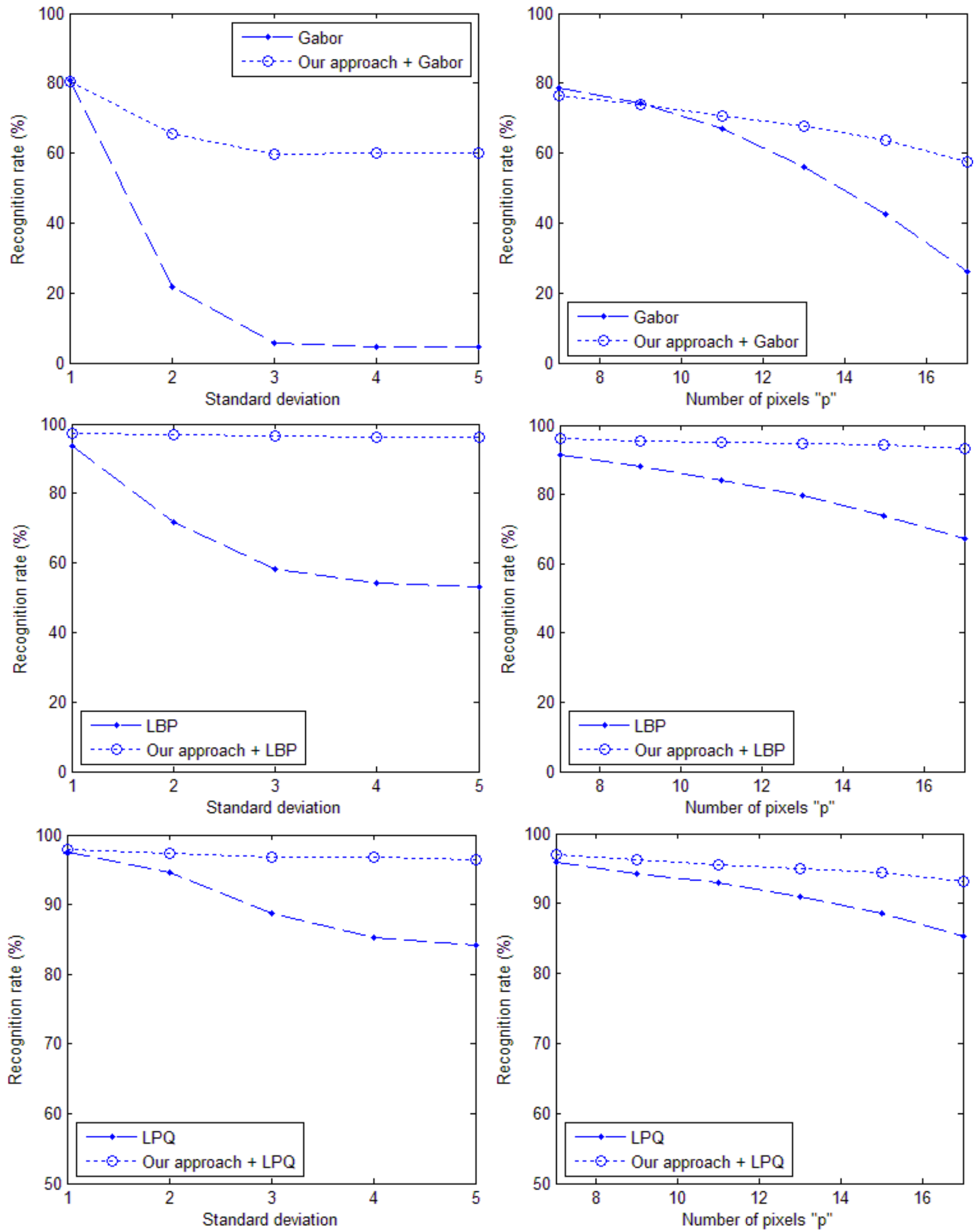

Figure 4. Left row: recognition rate obtained with increasing standard deviation $s$ of the Gaussian filter $F g_{(11, s)}$. Results obtained by using Gabor wavelets (up), LBP (center) and LPQ (down) facial descriptors with and without our approach. Right row: recognition rate obtained with the Motion filter $F m_{(p, 0)}$ with increasing number of pixels $p$. Results obtained by using Gabor wavelet (up), LBP (center) and LPQ (down) facial descriptor with and without our approach. 
tasks. In fact, it can be a very efficient approach since the recognition algorithm uses image frequencies for task identification. Consequently, this approach is very generic and we believe that it should be applied on different types of degradation such as the blocking effect visible on highly compressed images. In a general way, we believe that a recognition algorithm should be improved as a measure of the degradation process can be done.

\section{REFERENCES}

[1] Zhao, W., Chellappa, R., Phillips, P. J., and Rosenfeld, A., "Face recognition: A literature survey," $A C M$ Comput. Surv. 35(4), 399-458 (2003).

[2] Banham, M. and Katsaggelos, A., "Digital image restoration," Signal Processing Magazine, IEEE 14(2), 24-41 (1997).

[3] Stainvas, I. and Intrator, N., "Blurred face recognition via a hybrid network architecture," in [Pattern Recognition, 2000. Proceedings. 15th International Conference on], 2, 805-808 (2000).

[4] Ahonen, T., Rahtu, E., Ojansivu, V., and Heikkil, J., "Recognition of blurred faces using local phase quantization.," in $[I C P R], 1-4$ (2008).

[5] Ojansivu, V. and Heikkilä, J., "Blur insensitive texture classification using local phase quantization," in [ICISP '08: Proceedings of the 3rd international conference on Image and Signal Processing], 236-243, Springer-Verlag, Berlin, Heidelberg (2008).

[6] Crété-Roffet, F., Dolmiere, T., Ladret, P., and Nicolas, M., "The blur effect: Perception and estimation with a new no-reference perceptual blur metric," in [SPIE proceedings SPIE Electronic Imaging Symposium Conf Human Vision and Electronic Imaging], XII, EI 6492-16 (January 2007).

[7] Daugman, J. G., "Uncertainty relation for resolution in space, spatial frequency, and orientation optimized by two-dimensional visual cortical filters," Journal of the Optical Society of America A: Optics, Image Science, and Vision 2(7), 1160-1169 (1985).

[8] Liu, C. and Wechsler, H., "Gabor feature based classification using the enhanced fisher linear discriminant model for face recognition," Image Processing, IEEE Transactions on 11, 467-476 (April 2002).

[9] Shen, L. and Bai, L., "A review on gabor wavelets for face recognition," Pattern Anal. Appl. 9(2), 273-292 (2006).

[10] Ojala, T., Pietikainen, M., and Harwood, D., "A comparative study of texture measures with classification based on feature distributions," PR 29, 51-59 (January 1996).

[11] Ojala, T., Pietikainen, M., and Maenpaa, T., "Multiresolution gray-scale and rotation invariant texture classification with local binary patterns," Pattern Analysis and Machine Intelligence, IEEE Transactions on 24, 971-987 (July 2002).

[12] Ahonen, T., Hadid, A., and Pietikainen, M., "Face recognition with local binary patterns," in [Lecture Notes in Computer Science], Computer Vision - ECCV 2004 3021/2004, 469-481, Springer-Verlag, Berlin, Heidelberg (2004).

[13] Phillips, P., Moon, H., Rauss, P., and Rizvi, S., "The feret evaluation methodology for face-recognition algorithms," in [Computer Vision and Pattern Recognition, 1997. Proceedings., 1997 IEEE Computer Society Conference on], 137-143 (June 1997). 